\title{
Mild Keratoconus in the Mother of a Patient with Down Syndrome: Case Report and Clinical Hypothesis
}

\author{
1,2,3 Marcella Q Salomão, ${ }^{1}$ Ana L Höfling-Lima, ${ }^{2,4,5,6}$ Fernando Faria-Correia, ${ }^{2,7}$ Bernardo T Lopes, ${ }^{8}$ Daniel G Dawson, \\ ${ }_{1,2,3,9}$ Renato Ambrósio Jr
}

\begin{abstract}
The purpose of this study is to report a case of mild or forme fruste keratoconus (FFKC) in the mother of a patient with Down syndrome (DS) who presented for cataract surgery and to propose a possible association of mothers with mild forms of ectasia and higher risk for delivering babies with DS. Advanced corneal imaging including corneal topography, corneal tomography, and the integrated corneal tomography and biomechanical assessment allowed the identification of a mild form of keratoconus in both eyes of this patient, and the correct characterization of the corneal structure and whole optics of the eye were crucial for IOL selection and planning cataract surgery.
\end{abstract}

Keywords: Corneal tomography, Corneal topography, Down syndrome, Forme fruste keratoconus, Keratoconus.

\section{${ }^{1,2,3} \mathrm{PhD}$ Doctorate, Research Associate, Attendant, ${ }^{1}$ Professor, $2,4,5,6$ Research Associate, Attendant, Associate Professor, ${ }^{2,7}$ Research Associate, Engineer, ${ }^{8}$ Associate Professor, ${ }^{1,2,3,9}$ Affiliated Professor, Founder and Research Director, Director, Adjunct Professor \\ ${ }^{1,2,3}$ Department for Ophthalmology of the Federal University of Sao Paulo, Sao Paulo, Brazil, Rio de Janeiro, Corneal Tomography and Biomechanics Study Group, Instituto de Olhos Renato Ambrósio and VisareRIO, Rio de Janeiro, Brazil \\ ${ }^{1}$ Department for Ophthalmology of the Federal University of Sao Paulo, Sao Paulo, Brazil \\ ${ }^{2,4,5,6}$ Rio de Janeiro Corneal Tomography and Biomechanics Study Group, Department of Cornea and Refractive, Hospital de Braga, Braga, Portugal, School of Health Sciences, University of Minho, Braga, Portugal \\ ${ }^{2,7}$ Rio de Janeiro Corneal Tomography and Biomechanics} Study Group, University of Liverpool, Liverpook UK

${ }^{8}$ Department of Ophthalmology, The University of Florida, Gainesville, Florida

1,2,3,9 Department for Ophthalmology of the Federal University of Sao Paulo, Sao Paulo, Brazil, Rio de Janeiro Corneal Tomography and Biomechanics Study Group, Instituto de Olhos Renato Ambrósio and VisareRIO, Rio de Janeiro, Brazil, Department of Ophthalmology, Federal University of the State of Rio de Janeiro, Brazil

Corresponding Author: Renato Ambrósio Jr, Affiliated Professor, Founder and Research Director, Director, Adjunct Professor, Department for Ophthalmology of the Federal University of Sao Paulo, Sao Paulo, Brazil, Rio de Janeiro Corneal Tomography and Biomechanics Study Group, Instituto de Olhos Renato Ambrósio and VisareRIO, Rio de Janeiro, Brazil, Department of Ophthalmology, Federal University of the State of Rio de Janeiro, Brazil, e-mail: dr.renatoambrosio@ gmail.com
How to cite this article: Salomão $M Q$, Hoffling-Lima $A L$, Faria-Correia F, Lopes BT, Dawson DG, Ambrósio R Jr. Mild Keratoconus in the Mother of a Patient with Down Syndrome: Case Report and Clinical Hypothesis. Int J Kerat Ect Cor Dis 2018;7(2):115-120.

\section{Source of support: Nil}

Conflict of interest: Renato Ambrósio Jr is a consult of Oculus Optikgeräte $\mathrm{GmbH}$.

\section{INTRODUCTION}

Keratoconus (KC) and ectatic corneal diseases represent an important area of research and a true subspecialty in modern ophthalmology. This multifactorial and genetic heterogeneous bilateral ectatic corneal dystrophy has an incidence of approximately 1 per 2000 in the general population, with a presumed interaction between genetics, environmental and other factors. ${ }^{1-4}$ Prevalence of KC might vary between 8.8 and 54.5 per $100.000^{1,2,5,6}$ and between 50 and 230 per 100.000, depending on the diagnostic criteria used and the study's population ethnicity. ${ }^{1,7-9}$ Moderate and advanced stages of KC are promptly recognized by a trained physician, but the identification of milder or subclinical forms of $\mathrm{KC}$ can represent a challenge at times. ${ }^{10,11}$ The introduction of refractive surgery and the paradigm shift related to the development of new treatment modalities have boosted the need to recognize milder forms of the disease and identify inherent predisposition for ectasia progression. ${ }^{12-16}$ Advanced corneal imaging including corneal topography, tomography along with an assessment of biomechanical properties have a fundamental role in this appropriate characterization. ${ }^{17,18}$ However, additional applications for advanced corneal imaging, beyond the identification of forme fruste keratoconus and screening for ectasia risk, can be further explored. For example, the recognition of milder forms of keratoconus called forme fruste (FFKC) in family members of KC patients might be helpful in the study and characterization of familial KC.

Down syndrome (DS) was first described by John Langdon Down in 1866, ${ }^{19}$ and is associated with a condition defined as chromosomal nondisjunction, at chromosome 21. Considered one of the major causes of intellectual disability, DS has an estimated incidence of 1 in 1000 to 1 in 700 live births in the general population. ${ }^{20}$ Several ophthalmic disorders have been reported in DS patients including 
epicanthus, ectropion, blepharoconjunctivitis, nystagmus, strabismus, nasolacrimal duct obstruction, high myopia, and lens opacities. ${ }^{21,22}$ The association between Down syndrome and keratoconus is also well established, with a reported prevalence ranging from $0.5 \%$ to $15 \%$ (10-300fold that of the normal population). ${ }^{1,22-27}$ This relationship might be explained not only by the fact that DS patients are usually affected by collagen-related disorders ${ }^{2,3,28,29}$ but also due to a frequent habit of eye rubbing usually found in these patients. ${ }^{28}$ However, the association between $\mathrm{KC}$ or FFKC as a risk factor for a mother having a son with Down syndrome has never been investigated. The purpose of this study was to report a case of FFKC in a mother of a patient with Down syndrome who presented for cataract surgery and to hypothesize a possible association between mothers with FFKC or KC having a higher risk of delivering a baby with DS.

\section{CASE REPORT}

An 80-year- old female patient presented because of poor quality of vision for both distance and near activities. She had a previous diagnose of cataract in both eyes and was seeking for surgery with premium multifocal intraocular lens implantation. Her family history was remarkable for their daughter with Down syndrome with keratoconus, who had a successful triple procedure over ten years ago at this clinic. UDVA was CF $1 \mathrm{M}$ in both eyes and manifest refraction was $-4.25-1.25 \times 84$ in OD and $-4.50-1.50 \times$ 96 in OS, giving 20/30 and 20/40, respectively. Slit lamp examination exhibited and a grade 2 nuclear cataract in both eyes, along corneas within normality but with more evidenced corneal nerves. Intraocular pressure was 16 $\mathrm{mm} \mathrm{Hg}$ in OU. The dilated retinal exam was unremarkable in both eyes. Specular microscopy documented normal endothelial mosaic with central cell counting of 2,410 and 2,421 cells $/ \mathrm{mm}^{2}$ in OD and OS, respectively.
Potential macular visual acuity with the McIntyre's super pinhole was 20/20 in both eyes.

Placido-disk corneal topography was performed with the keratograph 5 and with rotating Sheimpflug tomography with Pentacam HR (Oculus GmbH; Wetzlar, Germany). Corneal front curvature maps derived from Placido and Scheimpflug presented with similar aspects. Figure 1 illustrates the front curvature axial maps from the Pentacam, with the Smolek/Klyce absolute 1.5 D scale, demonstrated a relatively regular pattern, with no asymmetry of inferior steepening in OU. Maximal keratometry (K Max) was 44.6 D and 45.1 D in OD and OS, respectively. Interestingly, the topometric keratoconus classification on Pentacam demonstrated a borderline keratoconus index (KI) value in OD of 1.07 and abnormal value, 1.69 in OS (Fig. 1). No topometric keratoconus classification (TKC) was detected OU. The Belin/Ambrósio enhanced ectasia display (BAD) ${ }^{30,31}$ from OD and OS demonstrated abnormal front and back elevation maps, along with abnormal deviation values, including Df, Db, Dt, and final D (Figs 2 and 3). The biomechanical assessment was performed with the Corvis ST (Oculus; Wetzlar, Germany) and the tomographic-biomechanical index $(\mathrm{TBI})^{18}$ was 0.68 in OD and 0.50 in OS (Figs 4 and 5).

The diagnosis of mild or forme fruste keratoconus was made in both eyes, based on the described findings. The patient was then advised for a bilateral monofocal aspheric IOL implantation (Zeiss Asphina 509 M), and the Haigis formula was selected for IOL calculation. Surgeries were performed with no intercurrences aiming for partial monovision (aim -0.6 D OD and plano OS). The patient recovered well and was very satisfied after the procedure. Ultimate uncorrected distance visual acuity was 20/70 J1 in OD and 20/25 J5 in OS. Final manifest refraction was $-1.25-1.00 \times 85$ in OD and plano $-0.50 \times$ 100 in OS, giving 20/20 in OU.

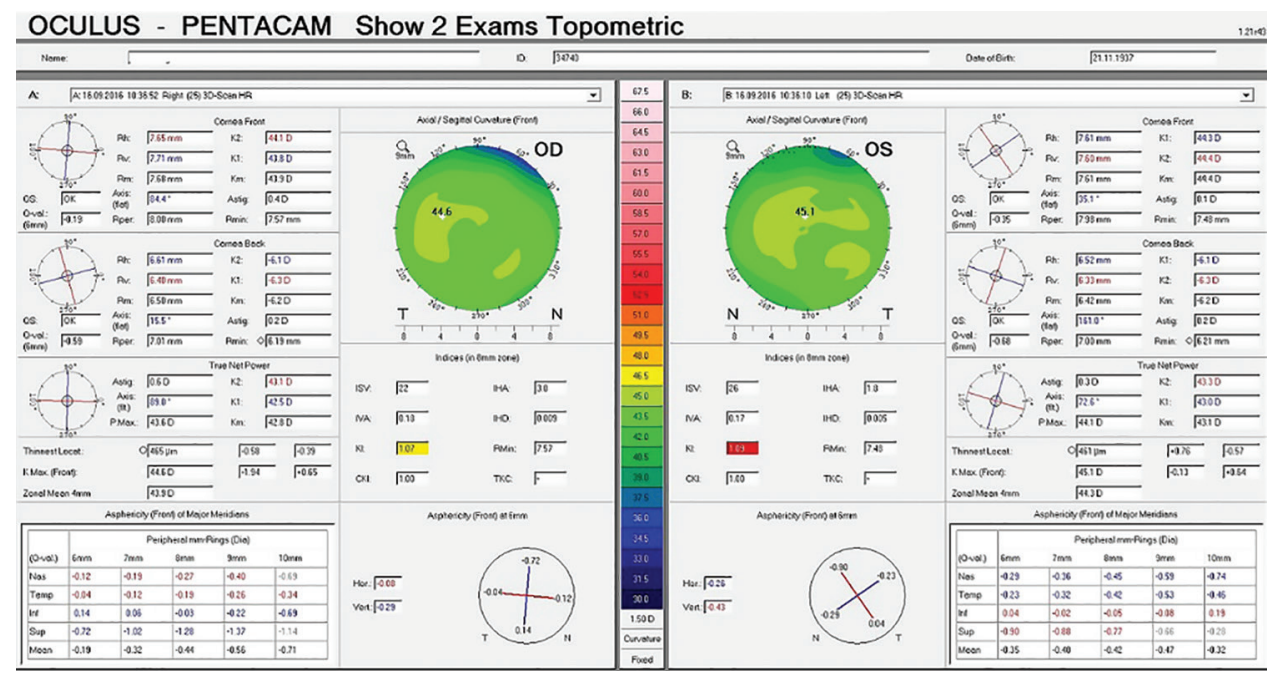

Fig. 1: Pentacam topometric axial maps from both eyes. No inferior-superior asymmetry was observed. Note topometric keratoconus classification on Pentacam which shows a borderline $\mathrm{KI}$ value in OD and abnormal value in OS 


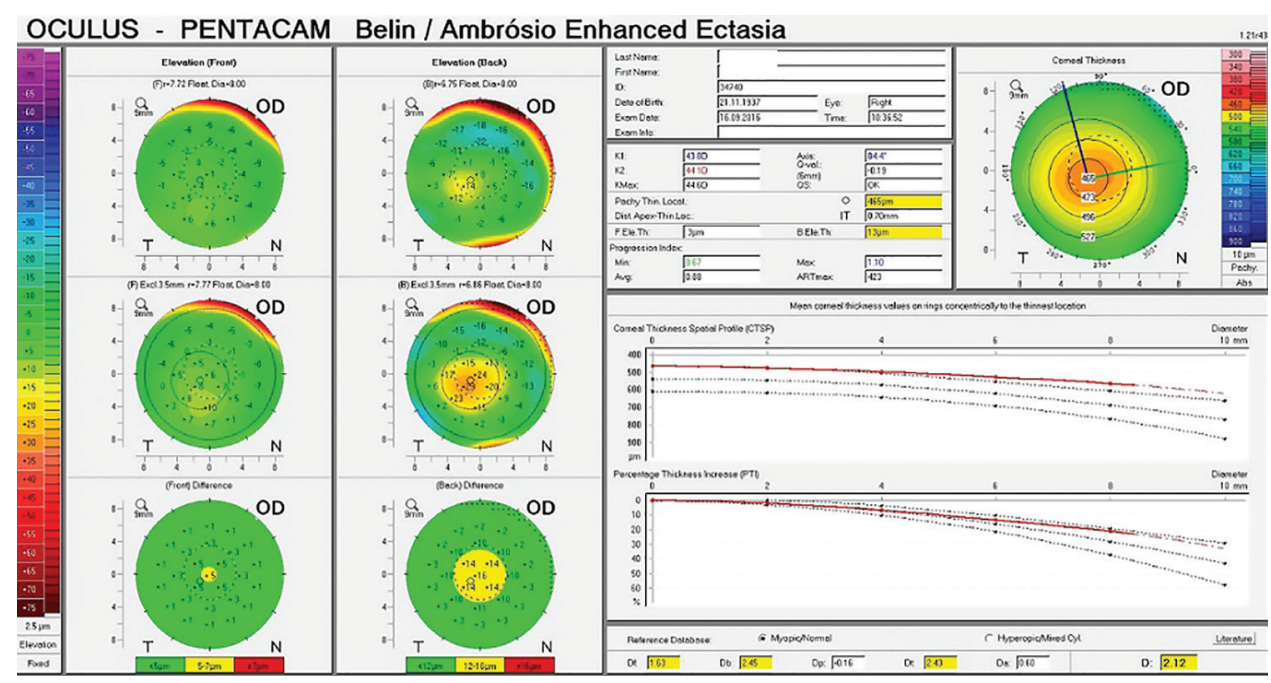

Fig. 2: Belin/Ambrósio enhanced ectasia display from the right eye. Note abnormal front and back elevation maps, along with abnormal deviation values, including $\mathrm{Df}, \mathrm{Db}, \mathrm{Dt}$, and final $\mathrm{D}$

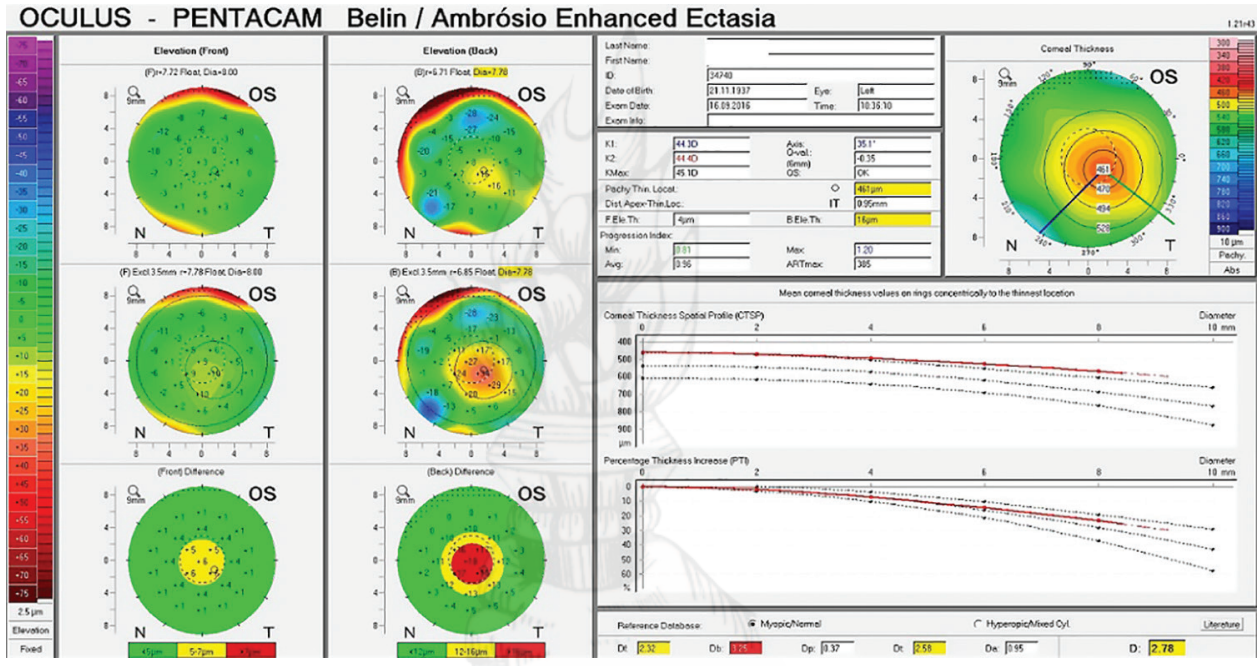

Fig. 3: Belin/Ambrósio enhanced ectasia display from the left eye

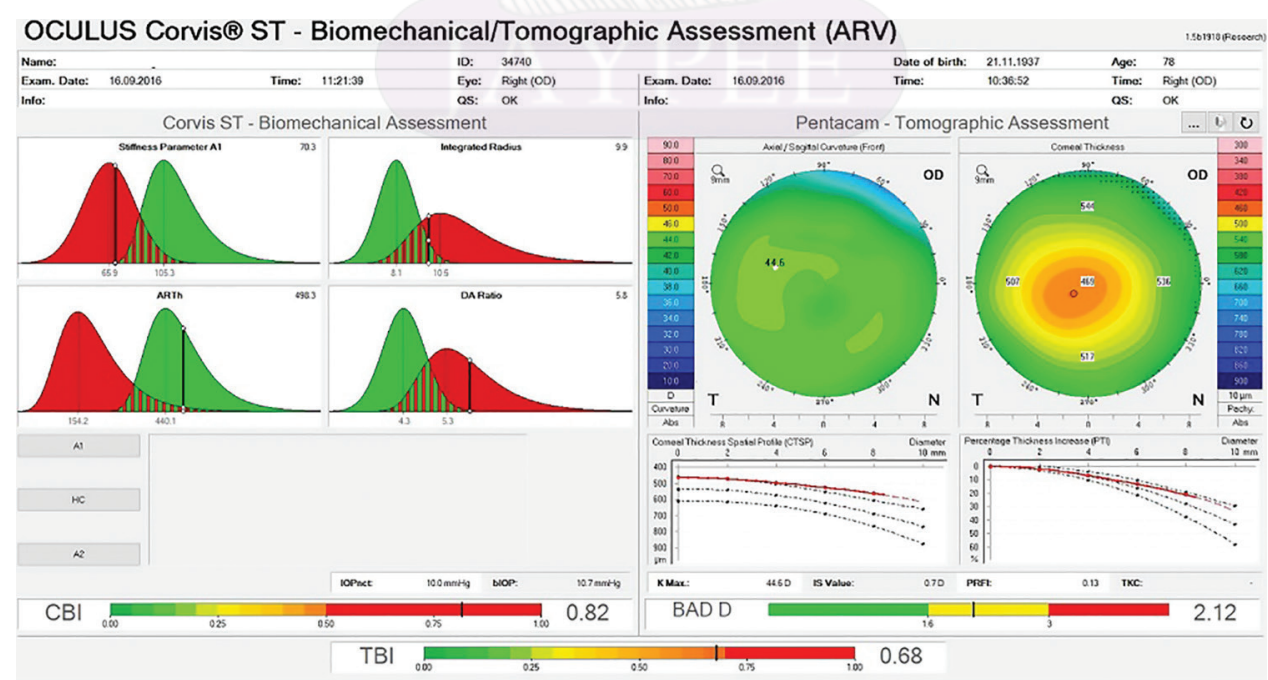

Fig. 4: Integrated corneal tomography and biomechanical display from Corvis ST and Pentacam with CBI and TBI from OD. Note an abnormal TBI value of 0.68

\section{DISCUSSION}

As the average population age is increasing, the number of cataract patients will also increase, and patients with
KC are more likely to develop cataracts. ${ }^{32,33}$ Corneal topography is an indispensable tool for investigating the anterior corneal surface and studies have demonstrated 


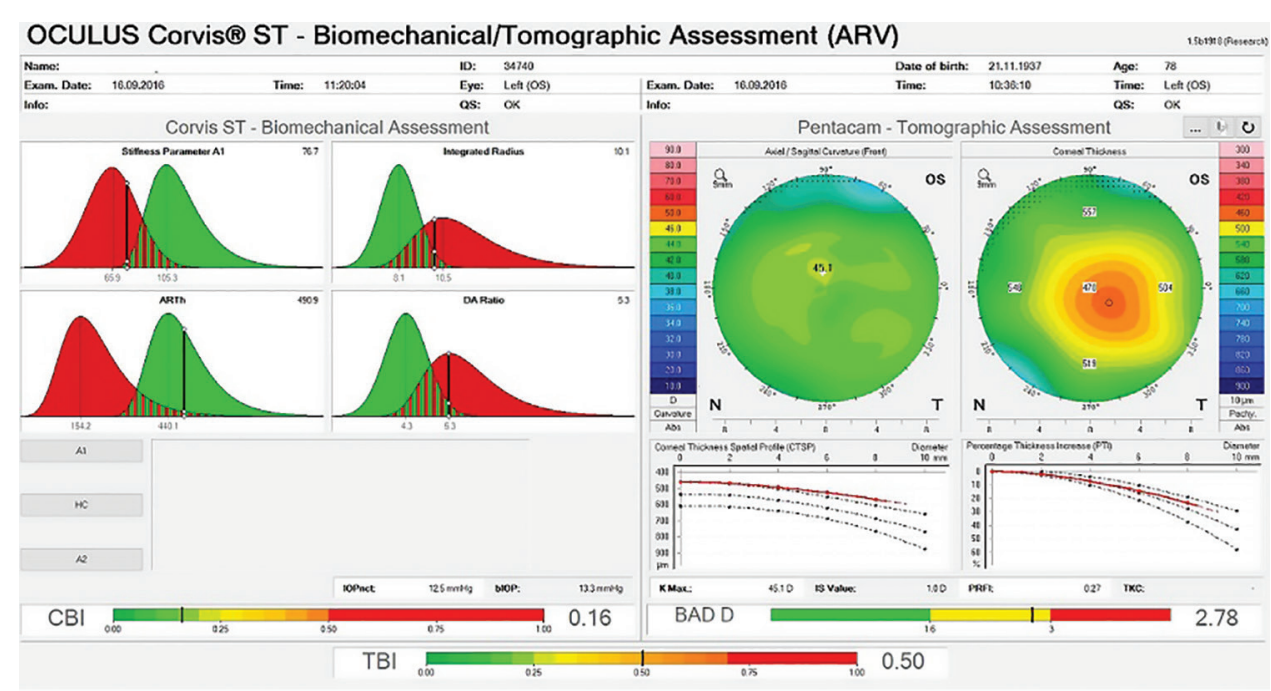

Fig. 5: Integrated corneal tomography and biomechanical display from Corvis ST and Pentacam with CBI and TBI from OS. Note an abnormal TBI value of 0.50

that this technology enhances the ability to detect the ectatic disease even before the development of slit lamp signs or loss of DCVA. ${ }^{34}$ Nevertheless, in recent years we witnessed a true revolution in corneal imaging with the emergence and development of new technologies capable of investigating not only the corneal shape but also clinical biomechanical behavior. This approach has been a critical tool in the investigation of keratoconus, and ectatic corneal diseases and studies have demonstrated the ability of these technologies to identify early forms of ectatic disease, even in cases undetected by Placido-based topography alone. ${ }^{35-40}$ This approach has special relevance when screening candidates for laser vision correction, but also when evaluating patients for cataract surgery.

In this case report, advanced diagnostic parameters derived from three-dimensional corneal tomography and biomechanical assessment were fundamental instruments for the identification of a mild form of keratoconus in both eyes. The correct preoperative characterization of the corneal structure was critical for the best IOL indication since patients with irregular corneal surfaces and significant higher order aberrations have poor outcomes with premium multifocal IOLs since these lenses divide light energy. ${ }^{33}$

Interestingly, different conditions have been proposed as FFKC, such as a normal topographic eye that naturally becomes ectatic over time, or the normal topographic eye of high or very asymmetric ectasia (VAE) cases. ${ }^{41}$ Suspect, incomplete and subclinical are additional terms proposed to refer to this condition, but modern corneal imaging with tomography and biomechanics has made them nonspecific and obsolete.

There is a consensus that both environmental factors and genetics play a role in the pathogenesis of keratoconus and ectatic corneal diseases. ${ }^{35,42}$ Despite extensive research, the genetic characterization in keratoconus is still challenging and different genes have been implicated in the development of the disease, including SOD1, VSX 1, miR-184, DOCK9, RAB3GAP1, and HGF. ${ }^{42}$ Although most KC patients appear to have a sporadic form of the disease, reports of familial $\mathrm{KC}$ are increasing, indicating that family members of $\mathrm{KC}$ patients have a higher risk to develop the disorder, and both autosomal dominant and recessive patterns might be implicated. ${ }^{42-46}$ Hopefully, the development of new genetic technologies such as whole exome or genome sequencing, might change our knowledge about the etiology of keratoconus, and even guide the therapeutic orientation. ${ }^{42}$

Down syndrome is related to trisomy of chromosome 21. Chromosomes are structures composed of DNA and proteins that present in every cell of the body and carry our genetic information. Humans normally have 46 chromosomes in each cell, divided into 23 pairs. Human cells undergo division in two possible ways-mitosis and meiosis. In the first method, one cell becomes two cells which have the same number and type of chromosomes as the parent cell. In the second method, which occurs for example in ovaries and testicles, one cell splits into two, with the resulting cells having half the number of chromosomes of the parent cell. In meiosis, there is an event called "disjunction", in which the pairs of chromosomes are supposed to split and merge to different spots in the dividing cell. However, many errors can occur in cell division and eventually, one pair does not divide and the whole pair goes to one spot. This is called "nondisjunction," and in this case, in the resulting cells, one will have 24 chromosomes and the other will have 22 chromosomes. In DS, $95 \%$ of cases are related to this situation in which one cell (from sperm or an egg) has two entire 21st chromosomes instead of one, so the resulting fertilized egg will have three 21st chromosomes. ${ }^{47}$ 
In the vast majority of the cases, the extrachromosomes are maternally derived, mainly due to non-disjunction in meiosis II (75\%) rather than meiosis I (25\%). ${ }^{47,48}$ Maternal age is also strongly associated with 21st chromosome trisomy, which is probably related to an increase in maternal meiotic nondisjunction.

We propose a possible association of mild forms of keratoconus as a potential risk factor along with older age, for mothers delivering babies with DS. Nevertheless, before any conclusion is taken, further studies are necessary to investigate the association of mild ectatic diseases as a risk factor, along with age, for a mother having a son with DS. Cohorts of mothers of patients with DS would probably represent an adequate study design to perform this investigation, and this needs to be done in different populations and in different countries.

\section{CONCLUSION}

In this case report, the mother of a patient with Down Syndrome and keratoconus presented for cataract surgery, seeking premium multifocal IOL implantation, Advanced corneal imaging allowed the detection of a mild form of ectasia in both eyes, which was a relative contraindication for multifocal IOL. The correct characterization of the corneal structure was fundamental for correctly choosing the intraocular lens model, as patients with corneal irregularities have frequently poor outcomes with multifocal IOLs. ${ }^{33}$ We recommend a detailed evaluation of the cornea before cataract surgery and advanced corneal imaging technologies have a relevant role on this investigation. We also propose a possible association of mild ectatic corneal disease as a risk factor along with age for mothers have a child with DS. Further studies, including cohorts of mothers of patients with DS, are necessary to explore this association.

\section{REFERENCES}

1. Loukovitis E, Sfakianakis K, Syrmakesi P, et al. Genetic Aspects of Keratoconus: A Literature Review Exploring Potential Genetic Contributions and Possible Genetic Relationships with Comorbidities. Ophthalmology and Therapy 2018.

2. Rabinowitz YS. Keratoconus. Survey of Ophthalmology 1998;42:297-319.

3. Kennedy RH, Bourne WM, Dyer JA. A 48-year clinical and epidemiologic study of keratoconus. American Journal of Ophthalmology 1986;101:267-273.

4. Krachmer JH, Feder RS, Belin MW. Keratoconus and related noninflammatory corneal thinning disorders. Survey of Ophthalmology 1984;28:293-322.

5. Belin MW, Duncan JK, Ambrósio Jr R, et al. A new tomographic method of grading keratoconus: the $\mathrm{ABCD}$ grading system. International Journal of Keratoconus and Ectatic Corneal Diseases 2015;4:85-93.

6. Mahmoud AM, Nunez MX, Blanco C, et al. Expanding the cone location and magnitude index to include corneal thickness and posterior surface information for the detection of keratoconus. American journal of ophthalmology 2013;156:1102-1111.

7. Alio JL, Pinero DP, Aleson A, et al. Keratoconus-integrated characterization considering anterior corneal aberrations, internal astigmatism, and corneal biomechanics. Journal of Cataract and Refractive Surgery 2011;37:552-568.

8. Barsam A, Petrushkin H, Brennan N, et al. Acute corneal hydrops in keratoconus: a national prospective study of incidence and management. Eye (London, England) 2015;29:469-474.

9. Pinero DP, Alio JL, Aleson A, et al. Corneal volume, pachymetry, and correlation of anterior and posterior corneal shape in subclinical and different stages of clinical keratoconus. Journal of Cataract and Refractive Surgery 2010;36:814-825.

10. Salomao M, Guerra F, Ramos IC, et al. Accuracy of topometric indices for distinguishing between keratoconus and normal corneas. International Journal of Keratoconus and Ectatic Corneal Diseases 2013;2:5.

11. Gatinel D SA. The Challenges of the Detection of Subclinical Keratoconus at Its Earliest Stage. International Journal of Keratoconus and Ectatic Corneal Diseases 2012;1:36-43.

12. Binder PS, Lindstrom RL, Stulting RD, et al. Keratoconus and corneal ectasia after LASIK. Journal of Cataract and Refractive Surgery 2005;31:2035-2038.

13. Binder PS. Ectasia after laser in situ keratomileusis. Journal of cataract and refractive surgery 2003;29:2419-2429.

14. Seiler T. The paradigm change in keratoconus therapy. Indian journal of ophthalmology 2013;61:381.

15. McGhee CN, Kim BZ, Wilson PJ. Contemporary Treatment Paradigms in Keratoconus. Cornea 2015;34 Suppl 10:S16-23.

16. Ambrosio R Jr F-CF, Silva-Lopes I, Azevedo- Wagner A, et al. Paradigms, paradoxes and controversies on Keratoconus and corneal ectatic diseases. International Journal of Keratoconus and Ectatic Corneal Diseases 2018;7:35-49.

17. Ambrosio R, Jr., Randleman JB. Screening for ectasia risk: what are we screening for and how should we screen for it? Journal of Refractive Surgery 2013;29:230-232.

18. Ambrósio R, Lopes BT, Faria-Correia F, et al. Integration of Scheimpflug-Based Corneal Tomography and Biomechanical Assessments for Enhancing Ectasia Detection. Journal of Refractive Surgery 2017;33:434-443.

19. Down JL. Observations on an ethnic classification of idiots. 1866. Mental Retardation 1995;33:54-56.

20. Vicic A, Hafner T, Bekavac Vlatkovic I, et al. Prenatal diagnosis of Down syndrome: A 13-year retrospective study. Taiwanese Journal of Obstetrics \& Gynecology 2017;56:731-735.

21. Kim JH, Hwang JM, Kim HJ, et al. Characteristic ocular findings in Asian children with Down syndrome. Eye (London, England) 2002;16:710-714.

22. Shapiro MB, France TD. The ocular features of Down's syn drome. American journal of ophthalmology 1985;99:659-663.

23. Little JA, Woodhouse JM, Saunders KJ. Corneal power and astigmatism in Down syndrome. Optometry and vision science: official publication of the American Academy of Optometry 2009;86:748-754.

24. Alio JL, Vega-Estrada A, Sanz P, et al. Corneal Morphologic Characteristics in Patients With Down Syndrome. JAMA ophthalmology 2018;136:971-978.

25. Hestnes A, Sand T, Fostad K. Ocular findings in Down's syndrome. Journal of Mental Deficiency Research 1991;35(Pt 3): 194-203. 
26. Van Splunder J, Stilma JS, Bernsen RM, et al. Prevalence of ocular diagnoses found on screening 1539 adults with intellectual disabilities. Ophthalmology 2004;111: 1457-1463.

27. Cullen JF, Butler HG. Mongolism (Down's Syndrome) And Keratoconus. The British Journal of Ophthalmology 1963;47:321-330

28. Zadnik K, Barr JT, Edrington TB, et al. Baseline findings in the Collaborative Longitudinal Evaluation of Keratoconus (CLEK) Study. Investigative ophthalmology \& visual science 1998;39:2537-2546.

29. Kenyon KR, Kidwell EJ. Corneal hydrops and keratoconus associated with mongolism. Archives of Ophthalmology (Chicago Ill : 1960) 1976;94:494-495.

30. Valbon BdF, Santos RT, Ramos I, et al. A tomografia de córnea e segmento anterior na propedêutica do exame complementar na avaliação de ectasia. Revista Brasileira de Oftalmologia 2013;72: 54-58.

31. Ambrosio R Jr, Ramos I, Lopes B, et al. Ectasia susceptibility before laser vision correction. Journal of cataract and refractive surgery 2015;41:1335-1336.

32. Moshirfar M, Walker BD, Birdsong OC. Cataract surgery in eyes with keratoconus: a review of the current literature. Current opinion in ophthalmology 2018;29:75-80.

33. Hallal R Jr, Ambrosio Jr R. the Importance of Screening for Ectatic Corneal Disease prior to Multifocal Intraocular Lens. International journal of keratoconus and ectatic corneal diseases 2018;7:1-6.

34. Ambrosio R Jr, Klyce SD, Wilson SE. Corneal topographic and pachymetric screening of keratorefractive patients. Journal of Refractive Surgery 2003;19:24-29.

35. Gomes JA, Tan D, Rapuano CJ, et al. Global consensus on keratoconus and ectatic diseases. Cornea 2015;34:359-369.

36. Ambrosio R Jr, Nogueira LP, Caldas DL, et al. Evaluation of corneal shape and biomechanics before LASIK. International ophthalmology clinics 2011;51:11-38.

37. Chan C, Ang M, Saad A, et al. Validation of an Objective Scoring System for Forme Fruste Keratoconus Detection and Post-
LASIK Ectasia Risk Assessment in Asian Eyes. Cornea 2015;34: 996-1004.

38. Saad A, Gatinel D. Validation of a new scoring system for the detection of early forme of keratoconus. International Journal of Keratoconus and Ectatic Corneal Diseases 2012;1: 100-108.

39. Ambrosio R Jr, Valbon BF, Faria-Correia F, et al. Scheimpflug imaging for laser refractive surgery. Current opinion in ophthalmology 2013;24:310-320.

40. Smadja D, Touboul D, Cohen A, et al. Detection of subclinical keratoconus using an automated decision tree classification. American journal of ophthalmology 2013;156:237-46.e1.

41. Klyce SD. Chasing the suspect: keratoconus. The British Journal of Ophthalmology 2009;93:845-847.

42. Wheeler J, Hauser MA, Afshari NA, et al. The Genetics of Keratoconus: A Review. Reproductive system \& sexual disorders: current research 2012.

43. Aknin C, Allart JF, Rouland JF. Unilateral keratoconus and mirror image in a pair of monozygotic twins. Journal Francais D'ophtalmologie 2007;30:899-902.

44. Weed $\mathrm{KH}$, MacEwen CJ, McGhee $\mathrm{CN}$. The variable expression of keratoconus within monozygotic twins: dundee University Scottish Keratoconus Study (DUSKS). Contact lens \& anterior eye. The Journal of the British Contact Lens Association 2006;29:123-126.

45. Abu-Amero KK, Kalantan H, Al-Muammar AM. Analysis of the VSX1 gene in keratoconus patients from Saudi Arabia. Molecular Vision 2011;17:667-672.

46. Stabuc-Silih M, Strazisar M, Ravnik-Glavac M, et al. Genetics and clinical characteristics of keratoconus. Acta dermatovenerologica Alpina, Pannonica, et Adriatica 2010;19:3-10.

47. Hernandez D, Fisher EM. Down syndrome genetics: unravelling a multifactorial disorder. Human Molecular Genetics 1996;5 Spec No:1411-1416.

48. Sherman SL, Petersen MB, Freeman SB, et al. Non-disjunction of chromosome 21 in maternal meiosis I: evidence for a maternal age-dependent mechanism involving reduced recombination. Human Molecular Genetics 1994;3:1529-1535. 